\title{
Skin condition and its relationship to systemic inflammation in chronic obstructive pulmonary disease
}

This article was published in the following Dove Press journal:

International Journal of COPD

II August 2017

Number of times this article has been viewed

\author{
Sebastian Majewski ${ }^{1,2}$ \\ Anna Pietrzak ${ }^{3}$ \\ Damian Tworek ${ }^{4}$ \\ Karolina Szewczyk ${ }^{5}$ \\ Anna Kumor-Kisielewskal \\ Zofia Kurmanowska ${ }^{5}$ \\ Paweł Górski',2 \\ Anna Zalewska-Janowska ${ }^{3, *}$ \\ Wojciech Jerzy \\ Piotrowski ${ }^{1,2, *}$ \\ 'Department of Pneumology \\ and Allergy, Medical University \\ of Lodz, Lodz, Poland; ${ }^{2}$ Healthy \\ Ageing Research Centre (HARC), \\ Lodz, Poland; ${ }^{3}$ Department of \\ Psychodermatology, Medical \\ University of Lodz, Lodz, Poland; \\ ${ }^{4}$ Department of General and \\ Oncological Pulmonology, Medical \\ University of Lodz, Lodz, Poland; \\ ${ }^{5}$ Department of Molecular Bases of \\ Medicine, Medical University of Lodz, \\ Lodz, Poland \\ *These authors contributed equally \\ to this work
}

\begin{abstract}
Background: The systemic (extrapulmonary) effects and comorbidities of chronic obstructive pulmonary disease (COPD) contribute substantially to its burden. The supposed link between COPD and its systemic effects on distal organs could be due to the low-grade systemic inflammation. The aim of this study was to investigate whether the systemic inflammation may influence the skin condition in COPD patients.
\end{abstract}

Materials and methods: Forty patients with confirmed diagnosis of COPD and a control group consisting of 30 healthy smokers and 20 healthy never-smokers were studied. Transepidermal water loss, stratum corneum hydration, skin sebum content, melanin index, erythema index, and skin temperature were measured with worldwide-acknowledged biophysical measuring methods at the volar forearm of all participants using a multifunctional skin physiology monitor. Biomarkers of systemic inflammation, including high-sensitivity C-reactive protein (hsCRP), interleukin-6 (IL-6), and tumor necrosis factor $\alpha$ (TNF- $\alpha$ ), were measured in serum using commercially available enzyme-linked immunosorbent assays.

Results: There were significant differences between COPD patients and healthy never-smokers in skin temperature, melanin index, sebum content, and hydration level $(P<0.05)$, but not for transepidermal water loss and erythema index. No significant difference was noted between COPD patients and smokers in any of the biophysical properties of the skin measured. The mean levels of hsCRP and IL-6 in serum were significantly higher in COPD patients and healthy smokers in comparison with healthy never-smokers. There were significant correlations between skin temperature and serum hsCRP $(R=0.40 ; P=0.02)$ as well as skin temperature and serum IL-6 $(R=0.49 ; P=0.005)$ in smokers. Stratum corneum hydration correlated significantly with serum TNF- $\alpha(R=0.37 ; P=0.01)$ in COPD patients.

Conclusion: Differences noted in several skin biophysical properties and biomarkers of systemic inflammation between COPD patients, smokers, and healthy never-smokers may suggest a possible link between smoking-driven, low-grade systemic inflammation, and the overall skin condition.

Keywords: chronic obstructive pulmonary disease, COPD, extrapulmonary manifestation, systemic inflammation, biophysical skin parameters, skin condition

\section{Introduction}

Chronic obstructive pulmonary disease (COPD) is a major cause of global morbidity and mortality and remains one of the greatest public health concerns. ${ }^{1}$ Many patients with COPD have concomitant chronic diseases linked to the same risk factors, mainly smoking and aging. In addition to the pulmonary features of COPD, many of its systemic effects have been well recognized. More than $30 \%$ of COPD patients 
have one additional chronic disease and another $40 \%$ have two or more comorbidities., ${ }^{2,3}$ Inflammatory mediators in the circulation (the "spill-over" theory) may contribute to skeletal muscle wasting, cachexia, and may initiate or worsen common COPD comorbidities such as ischemic heart disease, heart failure, osteoporosis, anemia, diabetes, and metabolic syndrome. These comorbidities result in impaired functional capacity, a decrease in quality of life, increased risk of hospitalizations, mortality, and significantly increased COPD-related healthcare costs. ${ }^{4,5}$ There is also a group of underrecognized COPD extrapulmonary manifestations, which include rhinosinusitis, dermatologic and ophthalmologic abnormalities, endocrinological disorders, or gastroesophageal reflux disease. These conditions may not always be fully recognized due to their relatively low clinical significance, data ambiguity, or inefficient mention in the literature. ${ }^{6}$

Skin is a multifunctional organ in the body acting as a protective physical barrier by absorbing UV radiation, preventing microorganism invasion, chemical penetration, and controlling the passage of water and electrolytes. In addition, skin has a major role in thermoregulation of the body as well as complex immunological, sensory, and autonomic functions. ${ }^{7}$

The data on skin condition in COPD are scarce. Studies confirm the association between smoking and premature aging of the skin. It has been shown that cigarette smoking is an independent risk factor for the development of premature facial wrinkling ${ }^{8-10}$ and that facial wrinkling is associated with COPD in smokers, and both disease processes may share a common susceptibility. ${ }^{11}$

The aim of this study was to compare the biophysical characteristics of the skin and serum levels of biomarkers of systemic inflammation in COPD subjects, healthy smokers, and never-smokers. In addition, we investigated whether skin condition in healthy smokers and COPD subjects is affected by systemic inflammation and whether it is linked to smoking.

\section{Materials and methods Study population}

A total of 90 volunteers including a cohort of 40 COPD patients, 30 healthy smokers with a minimum of 10 packyears history of smoking, and 20 healthy never-smokers were examined. This study was approved by the ethics committee of the Medical University of Lodz and was carried out according to the Declaration of Helsinki principles. All participants provided written informed consent before any study procedures were performed.

\section{Methods}

All study participants underwent clinical assessments including detailed medical history, physical examination, evaluation of biophysical skin variables, and spirometry. In addition, COPD subjects self-evaluated disease-specific symptoms with modified Medical Research Council (mMRC) dyspnea scale and the COPD Assessment Test (CAT). Physical capacity impairment was measured using the 6-minute walk test (6MWT). On the basis of available data, body mass index, airway obstruction, dyspnea, and exercise capacity (BODE) index for each of the COPD subjects was also calculated.

\section{Biophysical skin properties}

Transepidermal water loss (TEWL), stratum corneum hydration, skin sebum content, melanin index, erythema index, and skin temperature were measured at the volar forearm of all participants using a multifunctional skin physiology monitor (Courage \& Khazaka electronic GmbH, Cologne, Germany). This multiprobe adapter system consists of respective probes: TEWAmeter, corneometer, sebumeter, mexameter, and skin thermometer. The measurement of TEWL by TEWAmeter ${ }^{\circledR}$ TM 300 is based on the diffusion in an open chamber and is measured as $\mathrm{g} / \mathrm{m}^{2} / \mathrm{h} .{ }^{12}$ Corneometer $^{\circledR}$ CM 825 uses the high dielectric constant of water for analyzing the water-related changes in the electrical capacitance of the skin. It provides hydration measurements in systemspecific arbitrary units. ${ }^{13}$ Sebumeter ${ }^{\circledR}$ SM 815 uses the difference of light intensity through a plastic strip to indicate the amount of absorbed sebum. The sebum level is expressed in $\mu \mathrm{g} / \mathrm{cm}^{2} .{ }^{14}$ Mexameter ${ }^{\circledR} \mathrm{MX} 18$ calculates melanin index from the strength of the absorbed and the reflected light at 660 and $880 \mathrm{~nm}$, respectively, and similarly, erythema index at 568 and $660 \mathrm{~nm}$, respectively. ${ }^{15}$ Skin-Thermometer ST 500 was used to measure the skin temperature. The measurement is based on relative infrared temperature measurement.

All participants were asked to wash their hands with water only and not to use any soap or detergent or cosmetic products for at least 12 hours prior to measurements so that they would not affect measured biophysical skin parameters. Before skin measurements, participants rested for 30 minutes in a room with climate control having a temperature of $20^{\circ} \mathrm{C}-24^{\circ} \mathrm{C}$ and relative humidity of $30 \%-40 \%$ according to the recommendations of the manufacturer. The same trained investigator performed measurements of all skin variables between 8 a.m. and 12 p.m. to minimize time-dependent variations of skin biophysical characteristics. 


\section{Spirometry}

Spirometry assessment was performed using Lungtest 1000 spirometer (MES, Krakow, Poland) according to American Thoracic Society/European Respiratory Society guidelines. ${ }^{16}$ Postbronchodilator forced expiratory volume in 1 second $\left(\mathrm{FEV}_{1}\right)$, forced vital capacity (FVC), and $\mathrm{FEV}_{1} / \mathrm{FVC} \%$ were evaluated and recorded.

\section{The CAT}

CAT is a simple, validated health status instrument for patients with COPD. The self-administered questionnaire consists of eight items assessing various manifestations of COPD and global impact of the disease on health status. It is a simple quantified measure of health-related quality of life. Range of CAT scores is from 0 to 40 . A decrease in CAT score represents an improvement in health status, whereas an increase in CAT score represents a worsening in health status. ${ }^{17}$

\section{The mMRC dyspnea scale}

mMRC is a five-level rating scale based on the patient's perception of dyspnea in daily activities. It consists of five statements that describe the entire range of dyspnea from none (Grade 0 ) to almost complete incapacity (Grade 4). ${ }^{18}$

\section{The 6MWT}

The $6 \mathrm{MWT}$ is used for the evaluation of functional exercise capacity in patients with chronic respiratory diseases. In this study, 6MWT was performed using the methodology specified by the Polish Respiratory Society guidelines. ${ }^{19}$ Briefly, all COPD patients were instructed to walk as far as possible during 6 minutes. The $6 \mathrm{MWT}$ was performed in a flat, long, covered corridor which was $30 \mathrm{~m}$ long, meter-by-meter marked. When the test was finished, the distance covered by each was calculated.

\section{BODE index}

This multidimensional scoring system for COPD patients evaluates body mass index (BMI), measure of airflow obstruction ( $\mathrm{FEV}_{1} \%$ predicted), dyspnea score (grade in mMRC scale), and exercise capacity (distance covered in 6MWT). This composite marker of disease takes into consideration the systemic nature of COPD and is used to predict long-term outcomes in this population. ${ }^{20}$

\section{Biomarkers of systemic inflammation}

High-sensitivity C-reactive protein (hsCRP), interleukin-6 (IL-6), and tumor necrosis factor $\alpha$ (TNF- $\alpha$ ) were measured in serum using commercially available enzyme-linked immunosorbent assays (ELISA, R\&D Systems, Inc., Minneapolis, MN, USA).

\section{Statistical analysis}

Data were analyzed using GraphPad Prism 5 (GraphPad Software, Inc., La Jolla, CA, USA). Data were expressed as mean \pm standard error of mean (SEM) unless otherwise stated. Normality of data distribution was tested with Shapiro-Wilk test. Normally distributed data were analyzed using one-way analysis of variance (ANOVA) with a post hoc Tukey test. Non-normally distributed data were analyzed with Kruskal-Wallis test with the post hoc Dunn's test. Correlations were analyzed with Pearson correlation coefficient. Significance was accepted at $P<0.05$.

\section{Results Characteristics of participants}

Summary of characteristics of study participants is shown in Table 1. COPD patients were older than the control group and had greater mean smoking exposure than healthy smokers group. The mean time since diagnosis of COPD was $7.05 \pm 1.00$ years and mean $\mathrm{FEV}_{1}$ was $61.98 \% \pm 2.76 \%$ of predicted value. COPD patients were symptomatic with a mean CAT score of $15.8 \pm 1.18$ points.

Table I Characteristics of study participants

\begin{tabular}{|c|c|c|c|}
\hline Characteristics & $\begin{array}{l}\text { Never- } \\
\text { smokers }\end{array}$ & Smokers & COPD \\
\hline Number of subjects & 20 & 30 & 40 \\
\hline Sex (male/female) & $10 / 10$ & $11 / 19$ & $22 / 18$ \\
\hline Age (years) & $56.84 \pm 2.14^{*}$ & $55.97 \pm 1.72 *$ & $67.40 \pm 1.12$ \\
\hline Time since diagnosis (years) & N/A & N/A & $7.05 \pm 1.00$ \\
\hline $\begin{array}{l}\text { Smoking exposure } \\
\text { (pack-years) }\end{array}$ & N/A & $27.56 \pm 2.43 *$ & $41.99 \pm 2.78$ \\
\hline $\begin{array}{l}\text { Smoking status (current } \\
\text { smokers/ex-smokers) }\end{array}$ & $0 / 0$ & $30 / 0$ & $17 / 23$ \\
\hline $\mathrm{FEV}_{1}(\mathrm{l})$ & $3.06 \pm 0.13^{*}$ & $2.88 \pm 0.11 *$ & $1.52 \pm 0.07$ \\
\hline FEV (\% predicted) & $101.90 \pm 2.92 *$ & $96.62 \pm 4.0 I^{*}$ & $61.98 \pm 2.76$ \\
\hline $\mathrm{FEV} / \mathrm{FVC} \%$ & $77.69 \pm 1.43 *$ & $75.35 \pm 1.04 *$ & $52.49 \pm 1.57$ \\
\hline BMI $\left(\mathrm{kg} / \mathrm{m}^{2}\right)$ & $27.23 \pm 0.80$ & $27.72 \pm 0.68$ & $28.39 \pm 1.057$ \\
\hline 6MWT (m) & $\mathrm{N} / \mathrm{A}$ & $\mathrm{N} / \mathrm{A}$ & $388.6 \pm 11.08$ \\
\hline CAT (score) & N/A & N/A & $15.8 \pm 1.18$ \\
\hline mMRC (median, min-max) & N/A & N/A & I, 0-3 \\
\hline $\begin{array}{l}\text { BODE index } \\
\text { (median, min-max) }\end{array}$ & N/A & N/A & $1,0-6$ \\
\hline
\end{tabular}

Notes: $* P<0.001$ vs COPD. Data are expressed as mean \pm SEM or number. Abbreviations: 6MWT, 6-minute walk test; BMI, body mass index; BODE, BMI, airway obstruction, dyspnea, exercise capacity; CAT, COPD assessment test; COPD, chronic obstructive pulmonary disease; $\mathrm{FEV}_{1}$, forced expiratory volume in I second; FVC, forced vital capacity; mMRC, modified Medical Research Council dyspnea scale; N/A, not available; SEM, standard error of mean. 


\section{Biophysical skin properties}

The mean and standard error of mean (SEM) of measured biophysical skin parameters in all participants are shown in Figure 1. Skin temperature was significantly lower in healthy never-smokers $\left(29.46^{\circ} \mathrm{C} \pm 0.16^{\circ} \mathrm{C}\right)$ compared with healthy smokers $\left(30.18^{\circ} \mathrm{C} \pm 0.16^{\circ} \mathrm{C} ; P<0.01\right)$ and $\mathrm{COPD}$ subjects $\left(30.15^{\circ} \mathrm{C} \pm 0.13^{\circ} \mathrm{C} ; P<0.01\right)$. Stratum corneum hydration was higher in never-smokers $(43.60 \pm 0.97)$ compared with smokers $(38.35 \pm 1.66 ; P<0.05)$ and COPD subjects $(34.91 \pm 1.08 ; P<0.001)$. Mean skin melanin level was elevated in COPD subjects (150.2 \pm 4.95$)$ compared with never-smokers $(128.2 \pm 4.47 ; P<0.05)$. Mean sebum level was lower in COPD $(0.58 \pm 0.16)$ compared with never-smokers $(0.67 \pm 0.06 ; P<0.05)$. Erythema index and TEWL did not differ significantly between studied groups.

Correlations of biophysical skin variables with clinical measures

We found that in COPD subjects TEWL correlated negatively with the number of pack-years $(R=-0.33 ; P=0.03)$, time since diagnosis $(R=-0.31 ; P=0.04)$, and BODE index $(R=-0.35$; $P=0.02)$. Skin temperature correlated positively with BODE index $(R=0.36 ; P=0.02)$ while erythema index correlated negatively with $\mathrm{BMI}(R=-0.46 ; P=0.002)$ in COPD group
A

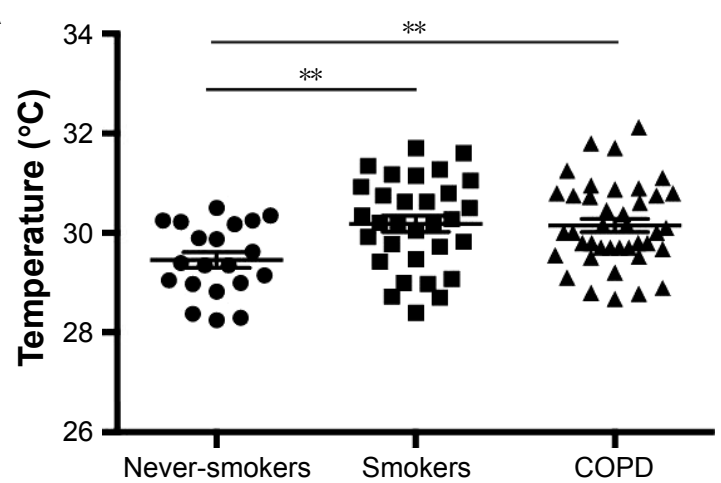

C

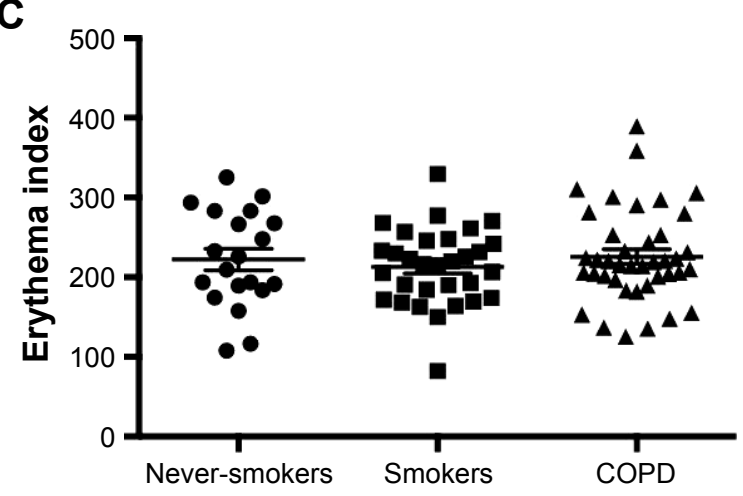

$\mathbf{E}$

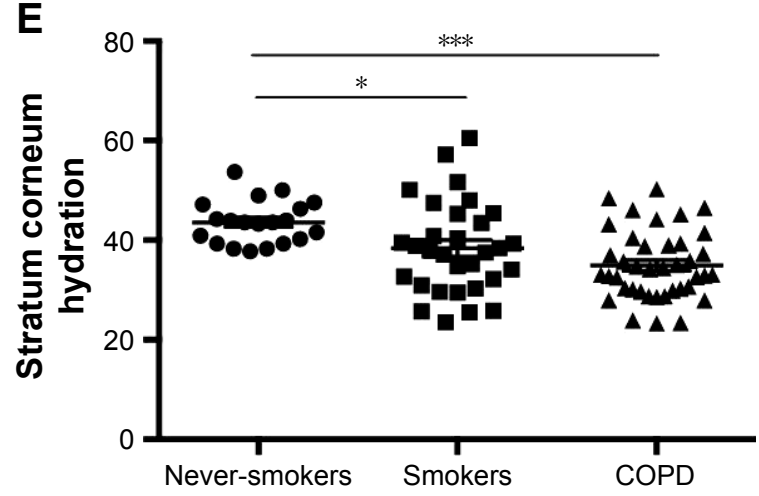

B

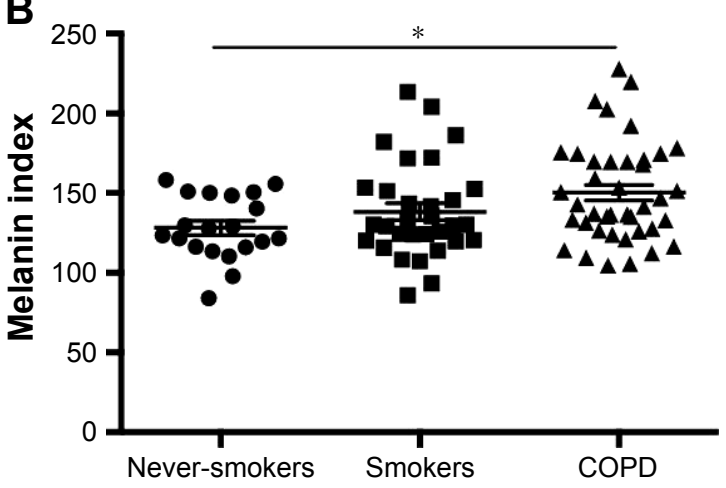

D

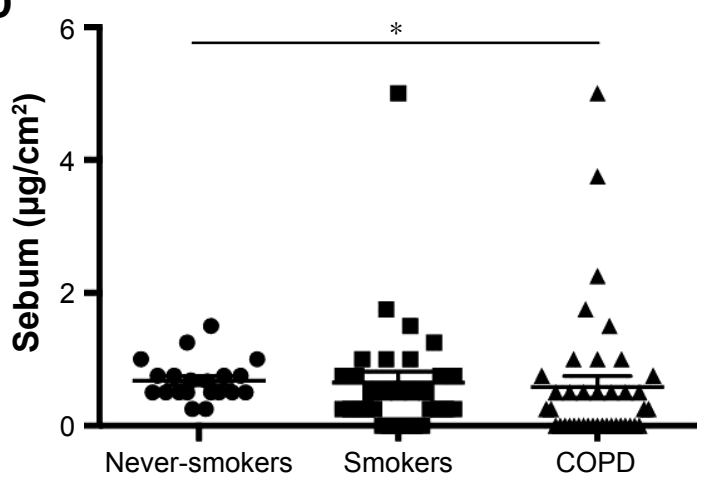

$\mathbf{F}$

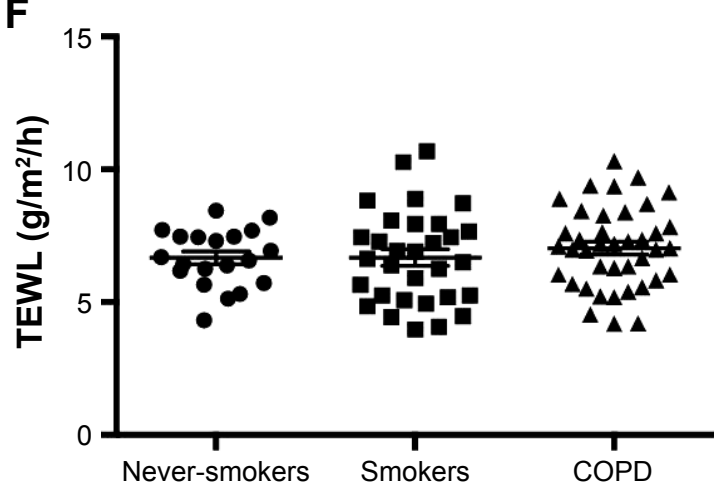

Figure I Biophysical skin parameters in never-smokers, smokers, and COPD subjects.

Notes: Panels showing measured biophysical skin parameters: (A) skin temperature, (B) melanin index, (C) erythema index, (D) sebum, (E) stratum corneum hydration, and (F) TEWL. $* P<0.05 ; * * P<0.01$; $* * * P<0.001$.

Abbreviations: COPD, chronic obstructive pulmonary disease; TEWL, transepidermal water loss. 
Table 2 Correlations of biophysical skin variables with clinical measures in COPD subjects

\begin{tabular}{lllllll}
\hline Clinical measures & Temperature & Melanin & Erythema & Sebum & Hydration & TEWL \\
\hline Pack-years & $R=-0.12 ; P=0.43$ & $R=0.14 ; P=0.38$ & $R=-0.00 ; P=0.99$ & $R=0.20 ; P=0.20$ & $R=0.27 ; P=0.08$ & $R=-0.33 ; P=0.03$ \\
Time since diagnosis & $R=0.25 ; P=0.11$ & $R=0.15 ; P=0.33$ & $R=0.07 ; P=0.66$ & $R=-0.05 ; P=0.74$ & $R=0.14 ; P=0.37$ & $R=-0.31 ; P=0.04$ \\
FEV $\%$ & $R=-0.22 ; P=0.15$ & $R=-0.06 ; P=0.71$ & $R=0.03 ; P=0.8 I$ & $R=-0.22 ; P=0.15$ & $R=0.17 ; P=0.27$ & $R=0.13 ; P=0.39$ \\
BMI & $R=-0.01 ; P=0.93$ & $R=-0.17 ; P=0.26$ & $R=-0.46 ; P=0.002$ & $R=-0.06 ; P=0.7$ & $R=-0.11 ; P=0.46$ & $R=-0.17 ; P=0.27$ \\
CAT & $R=0.10 ; P=0.52$ & $R=0.09 ; P=0.57$ & $R=-0.04 ; P=0.76$ & $R=-0.03 ; P=0.8 I$ & $R=-0.07 ; P=0.66$ & $R=-0.22 ; P=0.17$ \\
mMRC & $R=0.11 ; P=0.48$ & $R=0.01 ; P=0.90$ & $R=0.06 ; P=0.69$ & $R=-0.17 ; P=0.29$ & $R=-0.22 ; P=0.15$ & $R=-0.23 ; P=0.13$ \\
6MWT & $R=0.00 ; P=0.95$ & $R=0.22 ; P=0.16$ & $R=0.13 ; P=0.40$ & $R=-0.00 ; P=0.99$ & $R=0.10 ; P=0.52$ & $R=0.15 ; P=0.33$ \\
BODE index & $R=0.36 ; P=0.02$ & $R=0.06 ; P=0.69$ & $R=-0.07 ; P=0.64$ & $R=0.13 ; P=0.42$ & $R=-0.13 ; P=0.40$ & $R=-0.35 ; P=0.02$ \\
\hline
\end{tabular}

Abbreviations: 6MWT, 6-minute walk test; BMI, body mass index; BODE, BMI, airway obstruction, dyspnea, exercise capacity; CAT, COPD assessment test; COPD, chronic obstructive pulmonary disease; FEV , forced expiratory volume in I second; mMRC, modified Medical Research Council dyspnea scale; TEWL, transepidermal water loss.

as shown in Table 2. None of the biophysical skin variables measured correlated with age in any of the groups studied (data not shown). The most relevant and significant correlations between biophysical skin variables and clinical measures in COPD subjects are shown in Figure 2.

\section{Biomarkers of systemic inflammation}

Serum hsCRP and IL-6 levels were significantly lower in never-smokers $(2,046 \pm 328.8 \mathrm{ng} / \mathrm{mL}$ and $0.85 \pm 0.2 \mathrm{pg} / \mathrm{mL}$, respectively) compared with smokers $(5,907 \pm 1,155 \mathrm{ng} / \mathrm{mL}$, $P<0.05$ and $2.54 \pm 0.35 \mathrm{pg} / \mathrm{mL}, P<0.01$, respectively) as well as COPD subjects $(14,715 \pm 4,780 \mathrm{ng} / \mathrm{mL}, P<0.001$ and $6.08 \pm 1.37 \mathrm{pg} / \mathrm{mL}, P<0.001$, respectively). There were no differences in TNF- $\alpha$ levels between studied groups.

\section{Correlations of biophysical skin variables with biomarkers of systemic inflammation}

We found significant correlations between skin temperature and serum hsCRP $(R=0.40 ; P=0.02)$ as well as skin temperature and serum IL-6 $(R=0.49 ; P=0.005)$ in smokers. Stratum corneum hydration correlated significantly with serum TNF- $\alpha(R=0.37 ; P=0.01)$ in COPD subjects. There were also significant negative correlations between erythema and serum IL-6 $(R=-0.36 ; P=0.04)$ in smokers as well as erythema and serum TNF- $\alpha(R=-0.27 ; P=0.08)$ in COPD subjects. All correlations of biophysical skin variables with biomarkers of systemic inflammation are shown in Table 3. The graphical presentation of the most relevant and significant correlations between biophysical skin variables and biomarkers of systemic inflammation in smokers is shown in Figure 2.

\section{Discussion}

The complex structure of human skin and its biophysical characteristics turn it into an effective first-line defense against exogenous factors and help maintain homeostasis. This role is played by the epidermal barrier in which the corneal layer of epidermis has a particularly important function to perform. The condition of the epidermal barrier depends on its properties such as the amount of sebum produced, epidermis hydration, TEWL, or skin surface $\mathrm{pH}^{21}$

To our knowledge, our study is the first to demonstrate that skin biophysical properties deteriorate in COPD compared with healthy subjects and this phenomenon is caused by systemic inflammation triggered by cigarette smoke.

\section{Skin temperature}

Skin temperature is an important physiological measure that can reflect the presence of illness or injury and provide insight into the localized interactions between the body and the external environment. The human body retains tight thermoregulatory control about a set temperature of $\sim 37^{\circ} \mathrm{C}$, with life-threatening complications arising with core temperature increases as small as $3^{\circ} \mathrm{C} .{ }^{22}$ It is well known that exogenous pyrogens stimulate response of cytokine cascade in circulation, which are endogenous mediators of fever. ${ }^{23}$ Among all cytokines that are measurable in blood during lipopolysaccharide (LPS)-induced fever, circulating levels of IL-6 show the best correlation with the febrile changes of body temperature. ${ }^{24,25}$ In our study, skin temperature was significantly higher in smokers and COPD patients when compared with never-smokers (Figure 1), and it was accompanied by increased hsCRP and IL-6 serum concentrations. However, these markers of systemic inflammation correlated positively with skin temperature in smokers only which may suggest that systemic inflammatory response affects skin condition even in the absence of lung injury. Nevertheless, BODE index, a composite marker of severity of COPD, also correlated with skin temperature indicating that skin condition deteriorates in COPD patients as the disease progresses..$^{20}$

\section{Melanin index}

Melanin is one of the pigments that determine the skin color. ${ }^{26}$ In our study, skin melanin index was significantly higher 

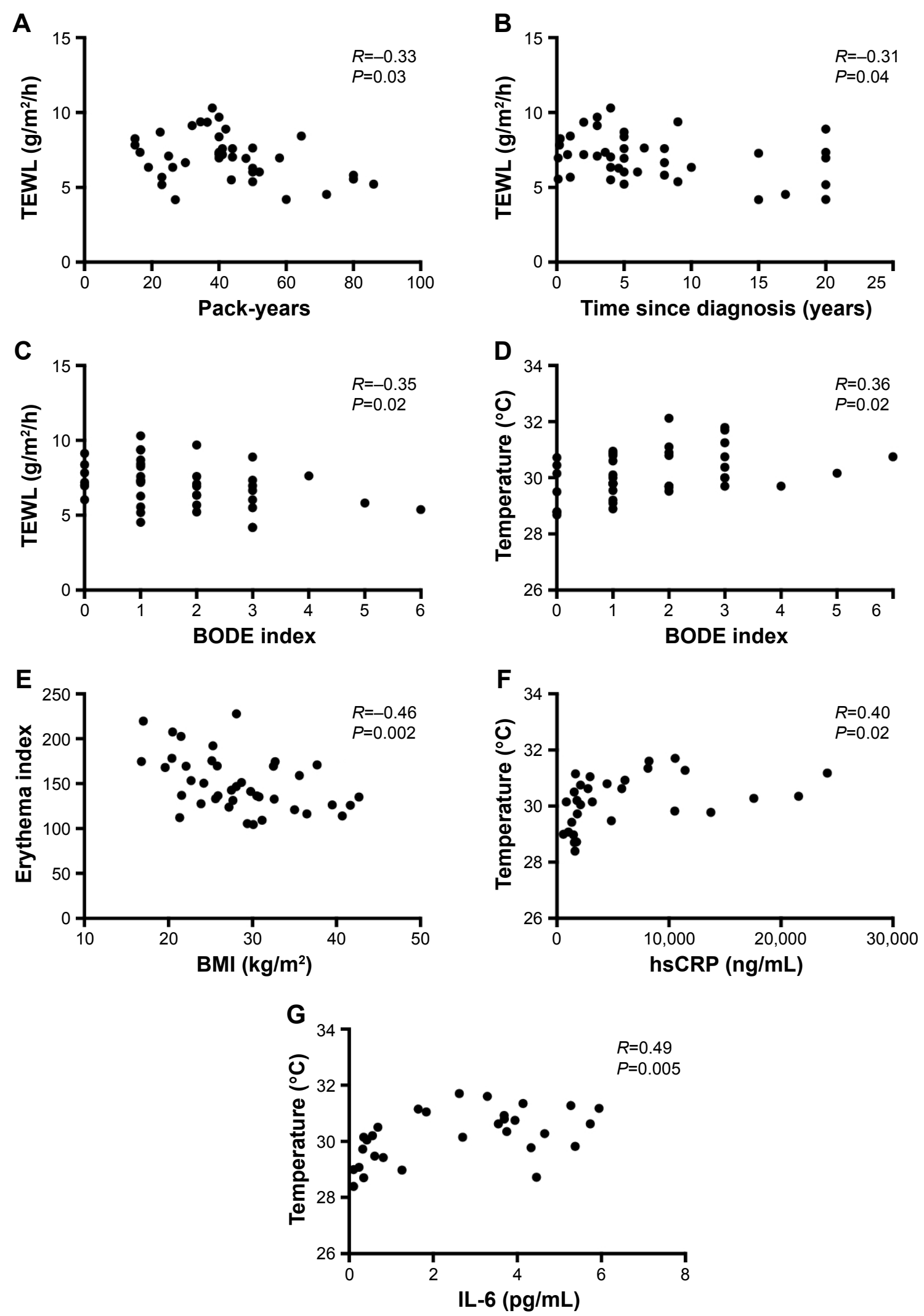

Figure 2 Graphical representation of the most relevant and significant correlations between biophysical skin variables and clinical measures in COPD subjects (A-E), and biomarkers of systemic inflammation in smokers ( $\mathbf{F}$ and $\mathbf{G})$.

Notes: Panels showing correlation between: (A) TEWL and pack-years in COPD subjects, (B) TEWL and time since diagnosis in COPD subjects, (C) TEWL and BODE index in COPD subjects, (D) skin temperature and BODE index in COPD subjects, (E) erythema and BMI in COPD subjects, (F) skin temperature and serum hsCRP in smokers, and (G) skin temperature and serum IL-6 in smokers.

Abbreviations: BMI, body mass index; BODE, BMI, airway obstruction, dyspnea, exercise capacity; COPD, chronic obstructive pulmonary disease; hsCRP, high-sensitivity C-reactive protein; IL-6, interleukin-6; TEWL, transepidermal water loss. 
Table 3 Correlations of biophysical skin variables with biomarkers of systemic inflammation

\begin{tabular}{lllllll}
\hline Serum biomarkers & Temperature & Melanin & Erythema & Sebum & Hydration & TEWL \\
\hline Never-smokers & & & & & & \\
hsCRP & $R=-0.27 ; P=0.24$ & $R=0.01 ; P=0.95$ & $R=0.12 ; P=0.58$ & $R=0.29 ; P=0.2$ & $R=0.3 ; P=0.18$ & $R=0.3 ; P=0.18$ \\
IL-6 & $R=-0.42 ; P=0.06$ & $R=-0.38 ; P=0.09$ & $R=0.11 ; P=0.09$ & $R=0.16 ; P=0.47$ & $R=0.12 ; P=0.59$ & $R=-0.01 ; P=0.95$ \\
TNF- $\alpha$ & $R=0.30 ; P=0.19$ & $R=0.22 ; P=0.35$ & $R=-0.11 ; P=0.62$ & $R=0.08 ; P=0.72$ & $R=-0.03 ; P=0.88$ & $R=0.24 ; P=0.30$ \\
Smokers & & & & & \\
hsCRP & $R=0.40 ; P=0.02$ & $R=-0.14 ; P=0.44$ & $R=-0.20 ; P=0.27$ & $R=0.31 ; P=0.09$ & $R=0.13 ; P=0.48$ & $R=-0.09 ; P=0.62$ \\
IL-6 & $R=0.49 ; P=0.005$ & $R=-0.02 ; P=0.89$ & $R=-0.36 ; P=0.04$ & $R=0.26 ; P=0.15$ & $R=-0.03 ; P=0.85$ & $R=-0.15 ; P=0.41$ \\
TNF- $\alpha$ & $R=0.2 ; P=0.26$ & $R=-0.09 ; P=0.61$ & $R=-0.05 ; P=0.79$ & $R=0.2 ; P=0.61$ & $R=-0.03 ; P=0.86$ & $R=0.22 ; P=0.22$ \\
COPD & & & & & \\
hsCRP & $R=0.1 ; P=0.52$ & $R=-0.13 ; P=0.4$ & $R=-0.24 ; P=0.40$ & $R=0.02 ; P=0.85$ & $R=0.16 ; P=0.31$ & $R=0.01 ; P=0.91$ \\
IL-6 & $R=-0.21 ; P=0.18$ & $R=-0.02 ; P=0.86$ & $R=-0.03 ; P=0.83$ & $R=0.02 ; P=0.89$ & $R=0.09 ; P=0.56$ & $R=0.03 ; P=0.83$ \\
TNF- $\alpha$ & $R=-0.04 ; P=0.78$ & $R=-0.24 ; P=0.12$ & $R=-0.27 ; P=0.08$ & $R=0.01 ; P=0.92$ & $R=0.37 ; P=0.01$ & $R=-0.07 ; P=0.62$ \\
\hline
\end{tabular}

Abbreviations: COPD, chronic obstructive pulmonary disease; hsCRP, high-sensitivity C-reactive protein; IL-6, interleukin-6; TEWL, transepidermal water loss; TNF- $\alpha$, tumor necrosis factor $\alpha$.

in the COPD cohort when compared with never-smokers group. The most important factor influencing skin pigmentation is sun exposure. ${ }^{27}$ There was no observed correlation between melanin index and age of study participants, which is in agreement with previous studies. ${ }^{28}$ However, it cannot be ruled out that measurements of melanin index in study participants in different seasons are responsible for observed skin pigmentation variations.

\section{Erythema index}

Quantification of erythema and pigmentation is useful for the analysis of skin tests and management of skin diseases. ${ }^{15}$ Erythema is the most common presenting sign in patients with skin diseases. We found no significant differences in erythema index between the groups; however, erythema index correlated negatively with BMI in COPD subjects. Low BMI in COPD is associated with increased mortality and reduced health status, quality of life, and exercise capacity, independently of airflow limitation. ${ }^{29-31}$ Thus, increased skin erythema may reflect worse overall condition of COPD patients.

\section{Sebum}

Sebum forms a type of insulation against excessive humidity and variations of ambient temperature. ${ }^{32}$ In our study, skin sebum level was significantly higher in COPD subjects in comparison with never-smokers. We did not observe any correlation between sebum level and age in any of the study groups, which is in agreement with findings of previous studies examining the relationship between skin sebum and age. ${ }^{33,34}$ In contrast, in a report from Switzerland, skin sebum level has been shown to decrease with age. ${ }^{35}$ Recent data suggest that inflammatory mediators may play a more important role than previously realized in the pathogenesis of skin diseases characterized by excessive production of sebum, for instance, acne vulgaris. ${ }^{36}$ It is possible that increased levels of proinflammatory cytokines generated in COPD and other chronic inflammatory conditions may trigger sebaceous glands to produce excess sebum. Increased sebum production may also be induced by nicotine, which stimulates sebocyte proliferation and lipid production. ${ }^{37}$

\section{Stratum corneum hydration}

The integrity of the epidermal barrier protects the skin against the excessive loss of water and maintains the correct hydration of epidermis. We found that stratum corneum hydration was significantly reduced in smokers and COPD subjects in comparison with never-smokers. Similar to other skin variables measured in our study, we did not find a correlation between skin hydration and age in any of the studied populations. This is in line with findings of other investigations. ${ }^{28,33,35}$ However, another study report did find a significant relationship between skin hydration and age. ${ }^{34}$ Although there is a substantial body of evidence describing skin inflammation and processes that lead to its clinical manifestations, there are no data on immunologic activity that occurs in the absence of any visual inflammatory cues. It is feasible that inflammation itself may be able to induce a functional skin barrier dysfunction. ${ }^{38}$ Therefore, low-grade systemic inflammation and oxidative stress generated in smokers and of greater degree in COPD subjects may impair epidermal permeability barrier function resulting in lower levels of skin hydration. This is in contrast to recent findings in a population of Chinese smokers which demonstrated significantly higher stratum corneum hydration on the forehead in light smokers ( $<20$ cigarettes a day) than in nonsmokers. Authors of this research attribute a high skin hydration on sebaceous gland-enriched sites (eg, forehead) of smokers 
to increased sebum production induced by nicotine. ${ }^{39}$ In the same study, authors showed lower stratum corneum hydration on sebum-impoverished site (dorsal hand) of heavy smokers ( $\geq 20$ cigarettes a day). Variations of measurements of skin hydration in our study and the study performed in Chinese population may also be due to the site of assessment and ethnicity. All measurements in our study were done at the volar forearm, while authors of discussed study collected data on the dorsal hand, forehead, and cheek. Previous studies showed variations in several biophysical properties of the skin among different skin locations. ${ }^{34}$ However, considering forearm as a sebum-impoverished site, our findings seem to be concordant with those from Chinese population study. ${ }^{39}$

\section{TEWL}

Water loss through epidermis is described by TEWL value and affects the level of epidermis moisture. ${ }^{12}$ TEWL is a parameter reflecting the integrity of epidermis water layer and is a very sensitive indicator of the epidermis barrier damage. ${ }^{7}$ In our study, TEWL was not different among studied groups of subjects and age did not show any significant effect on TEWL. A negative correlation between age and TEWL has been reported in several studies. ${ }^{33,40,41}$ However, other studies found no correlation between these two parameters. ${ }^{34,42}$ No difference in TEWL between never-smokers, smokers, and COPD subjects noted in our study is concordant with findings from a recent study performed in heavy cigarette smokers in a Chinese population. ${ }^{39}$ While there were no differences in basal TEWL between smokers and nonsmokers, permeability barrier recovery (after barrier disruption) was delayed in heavy smokers in comparison to nonsmokers. Permeability barrier recovery rates correlated negatively with the extent of cigarettes consumption. These results indicate that while basal permeability barrier function remains unchanged, heavy cigarette smokers display altered epidermal permeability barrier homeostasis. The authors conclude that these findings suggest the pathogenic role of cigarette smoking in skin condition. ${ }^{39}$ We found that in COPD subjects, TEWL also correlated negatively with the cigarette consumption, time since disease diagnosis, and BODE index. It is plausible that higher TEWL levels at early stages of disease are due to better epidermal hydration, which becomes reduced as the disease progresses. However, in contrast to our results, others found higher basal TEWL levels in cigarette smokers than in nonsmokers. ${ }^{43}$ This discrepancy with our data could be attributed to measurements collected from a different body region. While Muizzuddin et al measured TEWL on the cheek, ${ }^{43}$ our cohort and subjects from Chinese population ${ }^{39}$ had TEWL assessed on the forearm.
This study has several limitations, the most important being the difference in age between COPD patients and healthy smokers and never-smokers. Physiological aging could be responsible for changes in skin biophysical parameters. Although available data on this subject can be conflicting, we cannot exclude that the age difference could have biased the results. However, in our study none of the biophysical skin variables correlated with age. Comorbidities and systemic treatments are also important factors that could possibly influence obtained results. Another limitation is a small sample size. Despite the aforementioned limitations, the novel nature of our results fills a void in the literature. Studies including larger, age-balanced patient cohorts are warranted to determine the extent of skin abnormalities in COPD.

\section{Conclusion}

Experimental evaluation of serum inflammatory cytokines in our study suggests that smoking itself leads to the development of systemic inflammation, which in turn affects skin functions, observed in smokers and COPD patients. This hypothesis is supported by the lack of difference between COPD patients and smokers in any of the examined biophysical properties of the skin.

\section{Acknowledgments}

The authors would like to acknowledge Sonu Sahni MD, for his excellent English language corrections. The abstract version of this paper was presented at the 113th Annual Conference of American Thoracic Society as a poster presentation with interim findings and the poster's abstract has been published. ${ }^{44}$

\section{Disclosure}

The authors report no conflicts of interest in this work.

\section{References}

1. Global Initiative for Chronic Obstructive Lung Disease [homepage]. Available from: http://goldcopd.org. Accessed June 21, 2017.

2. Agusti A, Sobradillo P, Celli B. Addressing the complexity of chronic obstructive pulmonary disease: from phenotypes and biomarkery to scale-free networks, systems biology, and P4 medicine. Am J Respir Crit Care Med. 2011;183(9):1129-1137.

3. Barnes PJ, Celli BR. Systemic manifestations and comorbidities of COPD. Eur Respir J. 2009;33(5):1165-1185.

4. Mannino DM, Thorn D, Swensen A, Holguin F. Prevalence and outcomes of diabetes, hypertension, and cardiovascular disease in chronic obstructive pulmonary disease. Eur Respir J. 2008;32(4):962-969.

5. Foster TS, Filler JD, Marton JP, Caloyeras JP, Russell MW, Menzin J. Assessment of the economic burden of COPD in the US: a review and synthesis of the literature. COPD. 2006;3(4):211-218.

6. Miłkowska-Dymanowska J, Białas AJ, Zalewska-Janowska A, Gorski P, Piotrowski WJ. Underrecognized comorbidities of chronic obstructive pulmonary disease. Int J Chron Obstruct Pulmon Dis. 2015;10: $1331-1341$. 
7. McGrath JA, Uitto J. Structure and functions of the skin. In: Griffiths C, Barker J, Bleiker T, Chalmers R \& Creamer D, editors. Rook's Textbook of Dermatology. 9th ed. Oxford, UK: Wiley-Blackwell; 2016. Available from: http://www.rooksdermatology.com/

8. Kadunce DP, Burr R, Gress R, Kanner R, Lyon JL, Zone JJ. Cigarette smoking: risk factor for premature facial wrinkling. Ann Intern Med. 1991;114(10):840-844.

9. Koh JS, Kang H, Choi SW, Kim HO. Cigarette smoking associated with premature facial wrinkling; image analysis of facial skin replicas. Int $J$ Dermatol. 2002;41(1):21-27.

10. Aizen E, Gilhar A. Smoking effect on skin wrinkling in the aged population. Int J Dermatol. 2001;40(7):431-433.

11. Patel BD, Loo WJ, Tasker AD, et al. Smoking related COPD and facial wrinkling: is there a common susceptibility? Thorax. 2006;61(7): 568-571.

12. Shah JH, Zhai H, Maibach HI. Comparative evaporimetry in man. Skin Res Technol. 2005;11(3):205-208.

13. Gerhardt LC, Strässle V, Lenz A, Spencer ND, Derler S. Influence of epidermal hydration on the friction of human skin against textiles. J R Soc Interface. 2008;5(28):1317-1328.

14. Pande SY, Misri R. Sebumeter. Indian J Dermatol Venereol Leprol. 2005;71(6):444-446.

15. Yamamoto T, Takiwaki H, Arase S, Ohshima H. Derivation and clinical application of special imaging by means of digital cameras and Image J freeware for quantification of erythema and pigmentation. Skin Res Technol. 2008;14(1):26-34.

16. Miller MR, Hankinson J, Brusasco V, et al. ATS/ERS Task Force: standardisation of spirometry. Eur Respir J. 2005;26(2):319-338.

17. Jones PW, Harding G, Berry P, Wiklund I, Chen WH, Kline Leidy N. Development and first validation of the COPD assessment test. Eur Respir J. 2009;34(3):648-654.

18. Mahler DA, Wells CK. Evaluation of clinical methods for rating dyspnea. Chest. 1988;93(3):580-586.

19. Przybyłowski T, Tomalak W, Siergiejko Z, et al. Polish respiratory society guidelines for the methodology and interpretation of the 6 minute walk test (6MWT). Pneumonol Alergol Pol. 2015;83(4):283-297.

20. Cote CG, Celli BR. BODE index: a new tool to stage and monitor progression of chronic obstructive pulmonary disease. Pneumonol Alergol Pol. 2009;77(3):305-313.

21. Boer M, Duchnik E, Maleszka R, Marchlewicz M. Structural and biophysical characteristics of human skin in maintaining proper epidermal barrier function. Postęy Dermatol Alergol. 2016;33(1):1-5.

22. Kenny GP, Jay O. Thermometry, calorimetry, and mean body temperature during heat stress. Compr Physiol. 2013;3(4):1689-1719.

23. Roth J, De Souza GE. Fever induction pathways: evidence from responses to systemic or local cytokine formation. Braz J Med Biol Res. 2001;34(3):301-314.

24. Roth J, Conn CA, Kluger MJ, Zeisberger E. Kinetics of systemic and intrahypothalamic IL-6 and tumor necrosis factor during endotoxin fever in the guinea pig. Am J Physiol. 1993;265(3 Pt 2):R653-R658.

25. LeMay LG, Vander AJ, Kluger MJ. Role of interleukin-6 in fever in the rat. Am J Physiol. 1990;258(3 Pt 2):R798-R803.

26. Stamatas GN, Zmudzka BZ, Kollias N, Beer JZ. Non-invasive measurements of skin pigmentation in situ. Pigment Cell Res. 2004;17(6): $618-626$.
27. Hillebrand GG, Miyamoto K, Schnell B, Ichihashi M, Shinkura R Akiba S. Quantitative evaluation of skin condition in an epidemiological survey of females living in northern versus southern Japan. J Dermatol Sci. 2001;27(Suppl 1):S42-S52.

28. Mayes AE, Murray PG, Gunn DA, et al. Ageing appearance in China: biophysical profile of facial skin and its relationship to perceived age. J Eur Acad Dermatol Venereol. 2010;24(3):341-348.

29. Landbo C, Prescott E, Lange P, Vestbo J, Almdal TP. Prognostic value of nutritional status in chronic obstructive pulmonary disease. $\mathrm{Am} \mathrm{J}$ Respir Crit Care Med. 1999;160(6):1856-1861.

30. Mostert R, Goris A, Weling-Scheepers C, Wouters EF, Schols AM. Tissue depletion and health related quality of life in patients with chronic obstructive pulmonary disease. Respir Med. 2000;94(9):859-867.

31. Hallin R, Janson C, Arnardottir RH, et al. Relation between physical capacity, nutritional status and systemic inflammation in COPD. Clin Respir J. 2011;5(3):136-142.

32. Zouboulis CC. Acne and sebaceous gland function. Clin Dermatol. 2004;22(5):360-366.

33. Wilhelm KP, Cua AB, Maibach HI. Skin aging. Effect on transepidermal water loss, stratum corneum hydration, skin surface $\mathrm{pH}$, and casual sebum content. Arch Dermatol. 1991;127(12):1806-1809.

34. Firooz A, Sadr B, Babakoohi S, et al. Variation of biophysical parameters of the skin with age, gender, and body region. ScientificWorldJournal. 2012;2012:386936.

35. Wendling PA, Dell'Acqua G. Skin biophysical properties of a population living in Valais, Switzerland. Skin Res Technol. 2003;9(4): 331-338.

36. Harvey A, Huynh TT. Inflammation and acne: putting the pieces together. J Drugs Dermatol. 2014;13(4):459-463.

37. Zouboulis CC, Baron JM, Böhm M, et al. Frontiers in sebaceous gland biology and pathology. Exp Dermatol. 2008;17(6):542-551.

38. Vestergaard C, Hvid M, Johansen C, Kemp K, Deleuran B, Deleuran M. Inflammation-induced alterations in the skin barrier function: implications in atopic dermatitis. Chem Immunol Allergy. 2012;96: 77-80.

39. Xin S, Ye L, Man G, Lv C, Elias PM, Man MQ. Heavy cigarette smokers in a Chinese population display a compromised permeability barrier. Biomed Res Int. 2016;2016:9704598.

40. Kobayashi H, Tagami H. Functional properties of the surface of the vermilion border of the lips are distinct from those of the facial skin. Br J Dermatol. 2004;150(3):563-567.

41. Lopez S, Le Fur I, Morizot F, Heuvin G, Guinot C, Tschachler E. Transepidermal water loss, temperature and sebum levels on women's facial skin follow characteristic patterns. Skin Res Technol. 2000; 6(1):31-36.

42. Marrakchi S, Maibach HI. Biophysical parameters of skin: map of human face, regional, and age-related differences. Contact Dermatitis. 2007;57(1):28-34.

43. Muizzuddin N, Marenus K, Vallon P, Maes D. Effects of cigarette smoke on skin. J Cosmet Sci. 1997;48(5):235-242.

44. Majewski S, Pietrzak A, Tworek D, et al. Is Skin Affected by Systemic Inflammation in Chronic Obstructive Pulmonary Disease? Am J Respir Crit Care Med. 2017;195:A1344. Available from: http://www.atsjournals.org/doi/abs/10.1164/ajrccm-conference.2017.195.1_MeetingAbstracts.A1344

International Journal of COPD

\section{Publish your work in this journal}

The International Journal of COPD is an international, peer-reviewed journal of therapeutics and pharmacology focusing on concise rapid reporting of clinical studies and reviews in COPD. Special focus is given to the pathophysiological processes underlying the disease, intervention programs, patient focused education, and self management protocols.

This journal is indexed on PubMed Central, MedLine and CAS. The manuscript management system is completely online and includes a very quick and fair peer-review system, which is all easy to use. Visit http://www.dovepress.com/testimonials.php to read real quotes from published authors. 